EPJ Web of Conferences 79, 01006 (2014)

DOI: $10.1051 /$ epjconf/20147901006

(C) Owned by the authors, published by EDP Sciences, 2014

\title{
A promising new energy source: The Brownian motion of nanoresonator arrays
}

\author{
Jean-Selva Rattinacannou ${ }^{\mathrm{a}}$
}

\begin{abstract}
It is proposed a device designed to extract the Brownian motion energy. Since Brownian motion is usually a whoolly disordered motion, the first step consists in putting it in order with resonators, the Brownian motion of which is organized. Brownian motion energy is so low, that a very large number of them is needed. Since the Brownian motion phases of a resonator set are random, the second step is to set in phase those resonators and then, to extract the overall energy. The nanoresonator double-array device satisfies these requirements, provided a high accuracy in the tuning of the resonator frequencies is achieved.
\end{abstract}

\section{Introduction}

The numerous problems currently brought up by fossil energies gave rise, in recent times, to the rapid growth of the renewable energies, such as solar energy, wind power, hydropower, geothermal energy, wave energy, tidal energy, etc.

In the field of energy harvesting from ambient vibrations, devices which harvest energy from the surroundings have been studied, to produce self-powered wireless electronics and sensors, without the help of any electrochemical battery. Among them, those which convert vibrations into electrical energy by piezoelectric transduction, deliver only a little quantity of energy, hardly or just sufficient to power small sensors [1-6].

This theoretical study shows how to increase the energy produced by these vibration-based devices, to such an extent that it could enter the realm of renewable energy. In order to reach this goal, it is necessary to change the nature of the harvested vibrational energy. Instead of hazardous energy-limited ambient vibrations, which will never supply enough power for this purpose, it is proposed to have basis on the more reliable Brownian motion [7], which never stops and does exist everywhere and at all times, in the special form of resonator Brownian motion.

How is it possible to harvest energy from Brownian motion? It is possible because a resonator has a very particular property: its Brownian motion is organized, although thermal agitation is completely disordered. This is the starting point of the project and the first stage of the device concept.

\footnotetext{
${ }^{a}$ Corresponding author: jsrtncn@free.fr
}

This is an Open Access article distributed under the terms of the Creative Commons Attribution License 4.0, which permits unrestricted use, distribution, and reproduction in any medium, provided the original work is properly cited. 


\section{Resonator Brownian motion}

The resonator Brownian motion is not the classic broken-line trajectory of a very small particle in the air. It is a vibration at its fundamental resonance frequency.

The Brownian motion of a mechanical resonator [8-25], related to a damped harmonic oscillator, is described by the following Langevin differential equation:

$$
m \frac{d^{2} x}{d t^{2}}+r \frac{d x}{d t}+k x=f_{t h}
$$

where $x$ : resonator displacement $/ t$ : time $/ m$ : resonator mass $/ r$ : resonator mechanical resistance $/ k$ : resonator spring constant $/ f_{t h}$ : fluctuating force due to thermal agitation, that drives the resonator. Its spectral density, $S_{f_{t h}}(\omega)$, is constant over all frequencies, corresponding to a broad band random process or white noise:

$$
S_{f_{t h}}(\omega)=4 k_{B} T r
$$

with $\omega$ : angular frequency $/ k_{B}$ : Boltzmann's constant $/ T$ : absolute temperature in Kelvin.

Relations for $r$ and $k$ :

$$
r=\frac{m \omega_{0}}{Q}\left(\omega_{0}=2 \pi f_{0}\right) \quad k=m \omega_{0}^{2}
$$

where $f_{0}$ : resonator resonance frequency/ $\omega_{0}$ : resonator resonance angular frequency/ $Q$ : resonator quality factor.

In the frequency domain, the spectral density of $x, S_{x}(\omega)$, has the value [26]:

$$
S_{x}(\omega)=|H(\omega)|^{2} \cdot S_{f_{t h}}(\omega)
$$

with $H(\omega)$ : resonator frequency response complex function:

$$
|H(\omega)|^{2}=\frac{1}{\left(k-m \omega^{2}\right)^{2}+r^{2} \omega^{2}} .
$$

The function $S_{x}(\omega)$ is peaked at the resonance frequency $\omega_{0}$. Through the resonator frequency response function, the broad band random process of the thermal force is changed into a Gaussian narrow band random process [26], the resonator displacement, the time history of which looking like a sine wave of slowly varying amplitude and phase. So the Brownian motion of a resonator, while remaining an expression of thermal agitation is, at the same time, a quite constant in frequency vibration. This means that through a resonator, the anarchic and disorganized thermal energy of molecules is transformed into a regular motion, that is to say, into mechanical energy, and in our intention to harvest it, this is obviously of prime importance.

The mean amplitude of $x$ is equal to its standard deviation, $\sigma_{x}$, which is, since $\langle x\rangle$, the mean of $x$, is zero:

$$
\sigma_{x}=\left\langle x^{2}\right\rangle^{\frac{1}{2}}
$$

$\left\langle x^{2}\right\rangle$, the mean square of $x$, being also the integral of $S_{x}(\omega)$, taken over all frequencies [26]:

$$
\left\langle x^{2}\right\rangle=\int_{-\infty}^{+\infty} S_{x}(\omega) d \omega .
$$

The mean energy $E$ (both kinetic and elastic energy) of a mechanical resonator in equilibrium with a heat bath at temperature $T$ is [10]:

$$
E=\frac{1}{2} m\left\langle\left(\frac{d x}{d t}\right)^{2}\right\rangle+\frac{1}{2} k\left\langle x^{2}\right\rangle=k_{B} T .
$$


Table 1. Giving the mean Brownian motion amplitude of a PZT nanopillar (density: $8 \cdot 10^{3} \mathrm{Kg} / \mathrm{m}^{3}$ ) at $300^{\circ} \mathrm{K}$, where $e$ : pillar height $/ d$ : pillar diameter $/ V$ : pillar volume $/ m_{\text {eff }}$ : pillar effective mass (about a third of $m$ ), which replaces $m$ in equation (9), to calculate the right value of the mean amplitude (units are in SI).

\begin{tabular}{|c|c|c|c|c|c|c|}
\hline$e$ & $d$ & $f_{0}$ & $V$ & $m$ & $m_{\text {eff }}$ & $\sqrt{\left\langle x^{2}\right\rangle}$ \\
\hline $10^{-6}$ & $10^{-7}$ & $2 \cdot 10^{9}$ & $7.8 \cdot 10^{-21}$ & $6.2 \cdot 10^{-17}$ & $2.0 \cdot 10^{-17}$ & $1.1 \cdot 10^{-12}$ \\
\hline $10^{-7}$ & $10^{-7}$ & $2 \cdot 10^{10}$ & $7.8 \cdot 10^{-22}$ & $6.2 \cdot 10^{-18}$ & $2.0 \cdot 10^{-18}$ & $3.5 \cdot 10^{-13}$ \\
\hline $10^{-7}$ & $10^{-8}$ & $2 \cdot 10^{10}$ & $7.8 \cdot 10^{-24}$ & $6.2 \cdot 10^{-20}$ & $2.0 \cdot 10^{-20}$ & $3.5 \cdot 10^{-12}$ \\
\hline $10^{-8}$ & $10^{-8}$ & $2 \cdot 10^{11}$ & $7.8 \cdot 10^{-25}$ & $6.2 \cdot 10^{-21}$ & $2.0 \cdot 10^{-21}$ & $1.1 \cdot 10^{-12}$ \\
\hline
\end{tabular}

This energy is very low. In order to get a fair amount of energy, the only need is to counterbalance this low value. This is fulfilled with nanoresonator arrays.

According to the equipartition theorem, the average energy per degree of freedom is half the thermal energy:

$$
\frac{1}{2} k\left\langle x^{2}\right\rangle=\frac{1}{2} k_{B} T
$$

The mean amplitude of the resonator Brownian motion (table 1) is deduced from the foregoing:

$$
\sigma_{x}=\left(\frac{k_{B} T}{m \omega_{0}^{2}}\right)^{\frac{1}{2}} .
$$

From Table 1, it can be seen that pillar sizes must be small enough, so that the ratio vibration amplitude/pillar height is not too small, to ensure an electric signal will be detected at the pillar ends.

The following new reasoning completes the above well-known results on Brownian motion, and is used for the device working principle:

"Let us consider a system in equilibrium at temperature $T$, containing just one resonator surrounded by vacuum, the Brownian motion of the resonator being kept stopped by any means (for instance, by pressing on it the tip of an AFM). At a given time $t_{1}$, the resonator is released, free to vibrate, and the system is isolated from the exterior. After a certain time, at time $t_{2}$, the resonator Brownian motion has recovered its full amplitude, and the system is in thermodynamic equilibrium. Now, between the first state of the system, at time $t_{1}$, when the resonator is non-vibrating, and the second state, at time $t_{2}$, when it normally vibrates, energy is supplied to the resonator. But since the system is isolated, the law of energy conservation involves this energy supply comes from a decrease of the thermal energy of the system itself, that is to say, from a lowering of its temperature."

\section{Device description}

The device is a double-array, made up of two identical arrays of piezoelectric nanopillars, standing opposite the pillar tops, with a small gap between them [27].

\subsection{Pillar features}

Height: from 10 to 1000 nanometers (nm). Diameter: from 10 to $100 \mathrm{~nm}$. Aspect ratio (height/diameter ): from 1 to 10. An upper electrode, on the pillar tops, is what is left of the etching mask of a piezoelectric film. The piezoelectric orientation of the pillars is along their axis.

If the pillars are made of a ferroelectric material, the pillar polarization direction is the same on a whole array for all pillars, but is opposite on the other array, so that referred to the entire device, the polarizations of the two arrays have the same direction. 


\subsection{Array features}

Each array lies on a substrate such as a silicon wafer or a sapphire one. A bottom electrode, shared by the pillars, is deposited on the substrate. The arrays have a triangular lattice. Distance between two pillars, side to side: a tenth of the pillar height. Each array comprises a gigantic number of pillars: $10^{8}$, $10^{10}, 10^{12}$, or more, that is to say, for a square array with pillar diameter of $100 \mathrm{~nm}$ and pillar height of 1 micrometer, a side of about, respectively, 2, 20, and 200 millimeters $(\mathrm{mm})$. The gap between the pillar tops of the two arrays is approximately 10 times the pillar diameter. The pillar sizes of a double-array are strickly identical, making this latter particularly homogeneous.

\section{Working principle}

\subsection{Pillar vibration}

Nanopillars are tiny resonators, and their vibration is here a bulk wave one, directed along their axis, called "length mode". This mode has a resonance frequency in inverse ratio to the pillar height. By piezoelectricity, this mechanical oscillation makes electric charges appear at the pillar ends, creating between them an alternating electric potential difference.

In the conception of this device, the pillar oscillation is not due to the various vibrations prevailing in the surroundings, but is caused by thermal agitation, and therefore is called Brownian motion.

\subsection{Double- array}

All pillars of an array have the same resonance frequency, but random phases. The second stage of the device concept is to set in phase an entire array. This is the role of the double-array, which has, by this phase coherence, the ability to pool the energies of the pillars, and to make possible the energy harvesting.

\subsection{Setting in phase, and phase keeping}

\subsubsection{Hypothesis}

The practical faculty of setting in phase a pillar array is based on the following theoretical hypothesis, which expresses a to date never proposed property of resonator Brownian motion:

"It is possible to set in phase the random Brownian motions of a resonator set, and that these resonators still vibrate independently of each other, that is to say, the thermal agitation still acts individually and separately on the resonators, notably the mean amplitude of the resonator vibration is maintained."

This hypothesis is not so unlikely as it could seem at first sight, and in fact it is quite reasonable, since there are examples of spontaneously in phase thermal vibrations of molecules in some liquid crystals [28]. One explains the molecule shape leads to this collective or cooperative motion. Moreover, the vibration amplitude doesn't collapse with the collective motion. This fact is a strong argument in favour of this hypothesis, by taking these molecules to be small resonators.

Then, an array comprising a large number of in phase pillars does not behave like one single big mass or block with a negligible or imperceptible Brownian motion but on the contrary, each pillar keeps its own Brownian motion amplitude. 


\subsection{Procedure}

The importance of the gap (between the two arrays) must be here underlined. It should be greater than the pillar diameter, to avoid the direct coupling of each pillar of an array with its opposite of the other array, which would prevent the double-array from keeping the phase.

An alternating potential difference, above the usual one of the pillar Brownian motion, of an appropriate frequency is applied during a short time on the bottom electrodes, to set a single phase in the pillar individual potential differences (the permittivity of the pillars should not be too high, to ensure not too low a value of the electric field inside them, so that they will vibrate in phase). Once this outside action stops, the potential difference of the bottom electrodes decreases to reach its usual value. Then, each pillar contributes to the overall electric field that prevails between the opposite pillar tops of both arrays, and reciprocally, each pillar potential difference is inclined to stay in phase by this very field.

However, this inducement, this incentive, is weak and is only effective insofar as the pillar frequencies are tuned with a high accuracy. This accuracy is really the key to the functioning of the device. If it is achieved, there is self-keeping of the phase in the double-array.

\subsection{Energy extraction}

\subsubsection{Overall extraction}

In this case (phase keeping), the device produces an alternating potential difference between its bottom electrodes, ready for use, of the order of the one existing between the ends of a pillar, the electric charges at stake being proportional to the number of pillars in each array.

The pillar top is oscillating freely, and the upper electrode cannot be connected to any electric wire, which would cause immediately a strong damping of the vibration, because of the very tiny size of the pillars. This is a first difficulty. Secondly, there is a more basic impossibility which states that Brownian motion energy can't be directly and individually extracted from a nanopillar [29]. The previous difficulty and this impossibility are avoided by harvesting energy overall, from the whole double-array, at the device output.

Then, the double-array does not extract each pillar Brownian motion energy. Its purpose is to pool and to add, without any extraction, the low Brownian motion energies of a huge number of pillars, and this addition brings a lot of energy. So the energy of the double-array is no longer a Brownian motion energy, that is, a microscopic energy, but it is a macroscopic one, which behaves like any macroscopic energy, particularly, it can be extracted without any problem at the device output.

\subsubsection{Extraction cycle}

The energy extraction (i.e. the use of the device power) decreases the vibration of the pillars, breaking for that reason between them and the thermal energy, the thermodynamic equilibrium, and immediately activating, in order to restore it, the increase of this vibration, which consequently lowers the system temperature, because the pillar Brownian motion amplitude increase is made at the expense of the thermal energy.

The extraction cycle has two periods of equal duration:

- The extraction period: the pillar Brownian motion decreases.

- The restoring period (no extraction), or thermalization: the pillar Brownian motion increases.

The system comes back to equilibrium.

The extracted energy can progressively charge a capacitor or a battery, during the extraction periods of many cycles. The stored energy can be further used one way or another. The smaller the pillar size, then the bigger the pillar number, the higher the frequency, the larger the electric signal at the pillar ends, and 
the greater the harvested energy. Thus, there is a tremendous potential to develop by radically reducing the pillar size.

\subsubsection{Output power}

The energy that can be extracted from the double-array in one cycle is proportional to the pillar number $n$ in each array, and to $k_{B} T$. The output power is proportional to this energy, and is in inverse ratio to the cycle duration, which is governed by the relaxation time, or time constant $\tau$ :

$$
\tau=\frac{2 Q}{\omega_{0}} .
$$

Strictly, $\tau$ should be defined with $Q^{\prime}$, the double-array quality factor, but it is assumed the $Q^{\prime}$ value is close to the $Q$ one. The cycle duration is of the order of $2 \tau$, by taking the duration of each cycle period to be about $\tau$. So the power is proportional to $n, \omega_{0}, k_{B} T$, and $Q^{-1}$. If the pillar number $n$ and frequency $\omega_{0}$ are sufficiently high, the product $n \omega_{0}$, exceedingly large, will counterbalance the thermal energy value:

$$
n \omega_{0} \approx\left(k_{B} T\right)^{-1}
$$

Then, the output power will be of the order of $Q^{-1}$. If $Q=10^{2}$, the power will be $\approx 10^{-2}$ watt. Example: at room temperature, $k_{B} T=4 \times 10^{-21}$ joule; with $n=10^{10}$ and $f_{0}=20 \mathrm{GHz}, n \omega_{0}=1.2 \times 10^{21} \mathrm{rd} / \mathrm{s}$; with pillar height: $100 \mathrm{~nm}$ (frequency: about $20 \mathrm{GHz}$ ), diameter: $100 \mathrm{~nm}$, distance between to pillars, side to side: $100 \mathrm{~nm}$, the side of a square array of $10^{10}$ pillars is approximately $20 \mathrm{~mm}$, and the height of the double-array (with two wafers of thickness 300 microns) is about 602 microns. Such a device produces the above power value of $10^{-2}$ watt.

\section{Device fabrication}

An easy way to get an array of nanopillars, as identical as possible, is to etch a thin film, deposited on a substrate, with first a lithography process, followed by RIE or ICP [30-32]. Then the double-array is set up with a nanopositioning stage.

\section{Conclusion}

With the current situation of energy in the world, we can't afford not to take into account all the proposed solutions, and this theoretical study on a new device (the nanopillar double-array), which extracts energy from a new source (the Brownian motion), shows this device and this source are possible, provided the condition of a high accuracy in the pillar frequency tuning is satisfied. The device is feasible with the present technology, has low fabrication cost, its energy is greenhouse gas free, it doesn't contribute to the Earth's global warming, and therefore it should arouse the interest of researchers and physicists.

Experiments are now indispensable, and first of all to carry out prototypes of the device, in order to test the hypotheses made in the study, to validate the ideas which this device is conceived from, and to proove that Brownian motion can truly match the other renewable energy sources.

Piezoelectric MEMS (Micro Electro Mechanical Systems), or PiezoMEMS, are exactly the kind of structures needed to harvest the Brownian motion energy. But this device functions in the hyperfrequency range. So, in this undertaking, working closely together with hyperfrequency specialists is essential to succeed.

I warmly thank Miss Marisa Demko, English Professor, for checking the English of this paper. 
$3^{\text {rd }}$ European Energy Conference

\section{References}

[1] N. Elvin, A. Elvin, D. H. Choi, J. Strain Analysis 38, 115 (2003)

[2] S. R. Anton, H. A. Sodano, Smart Mater. Struct. 16, R1 (2007)

[3] J.-Q. Liu, H.-B. Fang, Z.-Y. Xu, X.-H. Mao, X.-C. Shen, D. Chen, H. Liao, B.-C. Cai, Microelectr. J. 39, 802 (2008)

[4] T. Galchev, H. Kim, K. Najafi, Procedia Chemistry 1, 1439 (2009)

[5] D. Zhou, N. Kong, D. S. Ha, D. J. Inman, Proc. of SPIE 7650, 765010 (2010)

[6] S. R. Anton, Multifunctional Piezoelectric Energy Harvesting Concepts, Ph.D. dissertation, State University, Blacksburg, VA (2011)

[7] R. Brown, Philosophical Magazine 4, 161 (1828)

[8] M. Morimura, K. Nakagawa, Y. Nezu, Jap. J. Appl. Phys. Suppl. 2, 135 (1974)

[9] V. B. Braginsky, A. B. Manukin, Measurement of Weak Forces in Physics Experiments (University of Chicago Press, Chicago, 1977)

[10] V. B. Braginsky, V. P. Mitrofanov, V. I. Panov, Systems with Small Dissipation (University of Chicago Press, Chicago, 1985)

[11] P. R. Saulson, Phys. Rev. D 42, 2437 (1990)

[12] O. D. Aguiar, W. W. Johnson, W. O. Hamilton, Rev. Sci. Instrum. 62, 2523 (1991)

[13] T. R. Albrecht, P. Grütter, D. Horne, D. Rugar, J. Appl. Phys. 69, 668 (1991)

[14] G. T. Gillies, R. C. Ritter, Rev. Sci. Instrum. 64, 283 (1993)

[15] T. B. Gabrielson, IEEE Trans. Electr. Devices 40, 903 (1993)

[16] C. T.-C. Nguyen, IEEE Trans. Ultrason. Ferroelectr. and Freq. Control 1, 489 (1995)

[17] G. I. González, P. R. Saulson, Phys. Lett. A 201, 12 (1995)

[18] G. I. González, Brownian Motion of a Torsion Pendulum Damped by Internal Friction, Ph.D. dissertation, Syracuse University, NY (1995)

[19] M. M. J. Treacy, T. W. Ebbesen, J. M. Gibson, Nature (London) 381, 678 (1996)

[20] E. Majorana, Y. Ogawa, Phys. Lett. A 233, 162 (1997)

[21] P. F. Cohadon, A. Heidmann, M. Pinard, Phys. Rev. Lett. 83, 3174 (1999)

[22] A. N. Cleland, M. L. Roukes, J. Appl. Phys. 92, 2758 (2002)

[23] R. Knobel, A. N. Cleland, Appl. Phys. Lett. 81, 2258 (2002)

[24] K. L. Ekinci, M. L. Roukes, Rev. Sci. Instrum. 76, 061101 (2005)

[25] J. C. Meyer, M. Paillet, S. Roth, Science 309, 1539 (2005)

[26] D. E. Newland, An Introduction to Random Vibrations, Spectral \& Wavelet Analysis (Third Edition, Dover Publications Inc., Mineola NY, 2005)

[27] J.-S. Rattinacannou, Piezoelectric Nanopillar Double-array Energy Harvesting Device, presented at the 3rd Int. PiezoMEMS Workshop, Washington DC, April 9-10 (2013) (unpublished)

[28] J. W. Goodby, Phase Structures of Calamitic Liquid Crystals, in Handbook of Liquid Crystals 2A, 12-13 (D. Demus Ed., Wiley, New York, 1998)

[29] D. P. Sheehan (University of San Diego, CA), private communication (January 2013)

[30] Y.-F. Chang, Q.-R. Chou, J.-Y. Lin, C.-H. Lee, Appl. Phys. A 86, 193 (2007)

[31] K. J. Morton, G. Nieberg, S. Bai, S. Y. Chou, Nanotechnology 19, 345301 (2008)

[32] M. D. Henry, ICP Etching of Silicon for Micro and Nanoscale Devices, Ph.D. dissertation, Caltech, CA (2010) 